\title{
Borotellurite glasses for gamma-ray shielding: an exploration of photon attenuation coefficients, structural, and thermal properties
}

\begin{abstract}
Gamma-ray attenuation characteristics and vibrational and thermal features have been studied for singly doped erbium (Er), dysprosium (Dy), and Er/Dycodoped sodium lithium zinc lead borotellurite glasses. For all glasses, the amorphous nature was confirmed from the $\mathrm{x}$-ray diffraction profiles, and $\mathrm{BO} 3, \mathrm{BO} 4, \mathrm{TeO} 4, \mathrm{TeO} 3+1$, and $\mathrm{TeO} 3$ structural units were identified by both Fourier transform infrared spectroscopy and Raman spectroscopy. Glass transition (Tg), onset crystallization (Tx), peak crystallization $(\mathrm{Tc})$, and melting $(\mathrm{Tm})$ temperatures including thermal stabilities (DT) were evaluated following the glass differential scanning calorimetry profiles. An enhancement in Tg (359 fi 399C) and DT variation at 131-169C with Er2O3, Dy2O3, and Er2O3/ Dy2O3 incorporation suggested that the prepared glasses possess good thermal stability. The radiation shielding properties within the $0.356-1.33-\mathrm{MeV}$ photon energy range were assessed for all the glasses. The mass attenuation coefficient (1/q) values have been calculated using Monte Carlo simulation code. Further, photon interaction parameters like effective atomic number (Zeff), half-value layer (HVL), and mean free path (MFP) were also computed. The host and 1.0 Er/1.0 Dy (mol.\%)codoped glasses possess the lowest and highest Zeff values and their magnitudes are varied within the range 11.40-15.99 and 12.14-17.26, respectively. For the host glass, exposure buildup factor values were calculated by the geometric progression (GP) fitting method within the $0.015-15-\mathrm{MeV}$ energy range and up to a penetration depth of $40 \mathrm{MFP}$. The removal cross sections RR ( $\mathrm{cm} 1)$ for fast neutrons were calculated to evaluate the attenuation of neutrons through the prepared glasses.
\end{abstract}

Keyword: Borotellurite glass; FTIR; Raman; DSC; Mass attenuation coefficient; Radiation shielding 